\title{
On Feedback Linearization for Robust Tracking Control of Flexible Joint Robots
}

\author{
Stig Moberg, Sven Hanssen \\ Division of Automatic Control \\ E-mail: stig@isy.liu.se, soha@kth.se
}

17th March 2008

Report no.: LiTH-ISY-R-2847

Accepted for publication in 17th IFAC World Congress, Seoul, Korea

Address:

Department of Electrical Engineering

Linköpings universitet

SE-581 83 Linköping, Sweden

WWW: http://www.control.isy.liu.se

AUTOMATIC CONTROL

REGLERTEKNIK

LINKÖPINGS UNIVERSITET

Technical reports from the Automatic Control group in Linköping are available from

http://www.control.isy.liu.se/publications. 


\begin{abstract}
Feedback linearization is one of the major academic approaches for controlling flexible joint robots. This contribution investigates the discrete-time implementation of the feedback linearization approach for a realistic threeaxis robot model. A simulation study of high speed tracking with model uncertainty is performed. It is assumed that full state measurements of the linearizing states are available. The feedback linearization approach is compared to a feedforward approach.
\end{abstract}

Keywords: robotics, manipulators, control, feedforward, feedback linearization, discrete-time, flexible joints 


\title{
On Feedback Linearization for Robust Tracking Control of Flexible Joint Robots
}

\author{
Stig Moberg ${ }^{*, * *}$ Sven Hanssen ${ }^{*, * * *}$ \\ * ABB AB - Robotics, SE-721 68 Västerås, Sweden. \\ ** Division of Automatic Control, Department of Electrical \\ Engineering, Linköping University, SE-581 83 Linköping, Sweden, \\ (e-mail: stig@isy.liu.se). \\ *** Department of Solid Mechanics, Royal Institute of Technology, \\ SE-100 44 Stockholm, Sweden, \\ (e-mail:soha@kth.se).
}

\begin{abstract}
Feedback linearization is one of the major academic approaches for controlling flexible joint robots. This contribution investigates the discrete-time implementation of the feedback linearization approach for a realistic three-axis robot model. A simulation study of high speed tracking with model uncertainty is performed. It is assumed that full state measurements of the linearizing states are available. The feedback linearization approach is compared to a feedforward approach.
\end{abstract}

Keywords: robotics, manipulators, control, feedforward, feedback linearization, discrete-time, flexible joints

\section{INTRODUCTION}

High accuracy control of industrial robot manipulators is a challenging task, which has been studied by academic and industrial researchers since the 1970's. The first approaches of linearizing a nonlinear system by nonlinear feedback can be found in the robotics literature from that decade. Control methods for rigid direct drive robots are, e.g., described in An et al. (1988). The two main approaches are feedforward control and feedback linearization, and both are based on a rigid dynamic model, combined with a diagonal PD or PID controller. Experimental evaluations are described in, e.g., Santibanez and Kelly (2001) and An et al. (1988). The conclusion in these is that feedforward control gives the same tracking performance as feedback linearization and that feedforward control is the preferred choice.

In Spong (1987) it is shown that flexible joint robots, i.e., elastic gear transmissions and rigid links, can be described by the so called simplified flexible joint model where the inertial coupling between the links and the motors are neglected. This approximation is valid for a reasonable high gear ratio. Furthermore, the viscous damping is also neglected in the simplified model. In the same article it is shown that the simplified flexible joint model can be linearized and decoupled by static feedback linearization, see also Spong et al. (2006).

In De Luca (1988) and De Luca and Lucibello (1998) it is shown that the complete flexible joint model can be linearized and decoupled by dynamic state feedback. A simulation study of feedback linearization using both the simplified and the complete flexible joint model is described in Nicosia and Tomei (1988), where it is claimed that the simplified model can cause large error in some operating conditions, although the gear ratio is high. The feedforward approach for flexible joint robots is described in, e.g., De Luca (2000). Experimental evaluations of con- trol methods for flexible joint robots are described in, e.g., Caccavale and Chiacchio (1994) (feedforward based on a rigid model), Jankowski and Van Brussel (1992) (discusses problems with feedback linearization due to its complexity and sample time requirement, suggests a simplified discrete-time predictive DAE approach with partial state feedback, based on a flexible joint model), Albu-Schäffer and Hirzinger (2000) (full-state feedback with gravity compensation based on a flexible joint model, concludes that feedback linearization is not possible to implement in available systems due to its complexity but also due to the requirements on model accuracy) and Thümmel et al. (2001) (feedforward based on a flexible joint model). In conclusion, the complete flexible joint feedback linearization is not implemented in any of these evaluations. No rigorous comparative simulation or experimental study of tracking with strict industrial requirements, using discretetime implementations of feedback linearization and feedforward control, for the flexible joint model, has been published to the authors' knowledge. However, simulation studies with reasonable realistic models are possible to perform.

This study is intended to help bridging the gap between theoretical nonlinear robot control and robot control practise. Many control methods suggested by researchers are seldom implemented in real systems and, on the other hand, many important control problems in the real world are not addressed in the academic research. The existence of such a gap in general control science is widely acknowledged and the need for a balance between theory and practise is expressed in, e.g., Åström (1994). From Bernstein (1999) we quote "I personally believe that the gap on the whole is large and warrants serious introspection by the research community". The same article also points out that the control practitioners must articulate their needs to the research community, and that motivating the researchers with problems from real applications "can 
have a significant impact on increasing the relevance of academic research to engineering practise". The problem is somewhat provocatively described in Ridgely and McFarland (1999) as, freely quoted, "what the industry in most cases do not want is stability proofs, guarantees of convergence and other purely analytical developments based on idealized and unrealistic assumptions".

In our simulation study, the real world is substituted with a simplified model, i.e., the (simplified) flexible joint model. Moreover, perfect state measurements are assumed. In a real implementation, some of the states would probably be estimated by means of an observer or simply by differentiation and low-pass filtering, disturbances and joint nonlinearities would be significant, and the real world elasticity would be much more complex than in our model, see, e.g., Wernholt and Moberg (2008). What we do assume, however, are important details such as a discrete implementation, continuous anti-alias filters, a reasonable requirement specification, realistic model parameters and small model parameter errors in our otherwise ideal model structure. Our rationale for this approach is that, if the chosen/evaluated control method does not prove its worth under these ideal circumstances, then the chances are small that a real implementation would give a different result. On the other hand, if the results are promising, then the next step would be to to increase the realism in the robot and sensor model, or to perform an experimental investigation.

\section{FLEXIBLE JOINT ROBOT MODEL}

In this section, a serial link flexible joint robot model is described. The model consists of a serial kinematic chain of $N$ rigid bodies. Rigid body $r b^{i}$ is described by its length $l^{i}$, mass $m^{i}$, center of mass $\xi^{i}$, and inertia tensor w.r.t. center of mass $J^{i}$. The rigid body $r b^{i}$ is connected to $r b^{i-1}$ by a torsional spring. The motors are placed on the preceding body and the inertial couplings between the motors and the rigid bodies are neglected under the assumption of high gear ratio, see, e.g., Spong (1987). The total system has $2 N$ DOF (i.e., the number of independent coordinates necessary to specify its configuration). The torque control of the motors are assumed to be ideal, so the $N$ input signals $u$ of the system are the motor torques. The equations of motion are derived by computing the linear and angular momentum and their time derivatives. By using Kane's method (Kane and Levinson, 1985; Lesser, $2000)$ the projected equations of motion are derived to yield a system of ordinary differential equations (ODE) with minimum number of DOF. The model equations can be described as a system of second order ODE's

$$
\begin{aligned}
M_{a}\left(q_{a}\right) \ddot{q}_{a}+n\left(q_{a}, \dot{q}_{a}\right)+K\left(q_{a}-q_{m}\right) & =0, \\
M_{m} \ddot{q}_{m}+K\left(q_{m}-q_{a}\right) & =u,
\end{aligned}
$$

where $\dot{x}$ denotes $d x / d t . M_{a}\left(q_{a}\right) \in R^{N \times N}$ is the inertia matrix for the links and $n\left(q_{a}, \dot{q}_{a}\right)=c\left(q_{a}, \dot{q}_{a}\right)+g\left(q_{a}\right)$, where $c\left(q_{a}, \dot{q}_{a}\right) \in R^{N}$ and $g\left(q_{a}\right) \in R^{N}$ describes the Coriolis, centrifugal and gravity torques. $M_{m} \in R^{N \times N}$ is the diagonal inertia matrix of the motors. The link and motor angular positions are denoted $q_{a} \in R^{N}$ and $q_{m} \in R^{N}$ respectively. Note that the gear ratio matrix, $r$, is not explicitly shown in the equations. All equations are expressed on the link side and $M_{m}=r^{T} M_{m}^{m} r$, where $M_{m}^{m}$ is the motor inertia matrix on the motor side.

For a complete model including the position and orientation of the tool, $Z$, the forward kinematic model of the

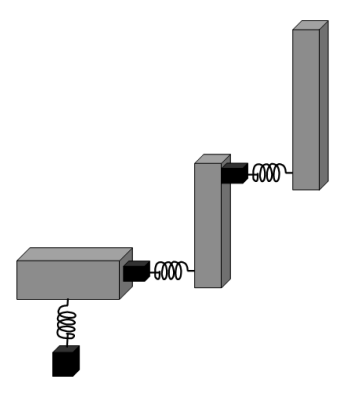

Fig. 1. A three axis flexible joint model

robot is needed. The kinematic model is a mapping of $q_{a} \in R^{N}$ to $Z \in R^{6}$. The complete model of the robot is then described by (1) and

$$
Z=\Gamma\left(q_{a}\right) \text {. }
$$

$\mathrm{Z}$ is the controlled output variable. The forward kinematics (2) is practically invertible, i.e., there are methods for handling singularities and multiple solutions (Sciavicco and Siciliano, 2000) to get the inverse kinematics

$$
q_{a}=\Gamma^{-1}(Z, C),
$$

where $C$ is a set of configuration parameters or other type of information, used to select a feasible solution. The link angular positions $q_{a}$ can thus be regarded as alternative output variables. A robot with $N=3$ is used in the simulation study to follow. This robot is illustrated in Figure 1.

\section{FEEDBACK LINEARIZATION AND FEEDFORWARD CONTROL OF A FLEXIBLE JOINT ROBOT}

The flexible joint robot is an example of a differentially flat system (Rouchon et al., 1993) which can be defined as a system where all state variables and control inputs can be expressed as an algebraic function of the desired trajectory for a flat output, and its derivatives up to a certain order. Feedback linearization by static or dynamic state feedback is equivalent to differential flatness (Nieuwstadt and Murray, 1998). By solving (1a) for $q_{m}$ and differentiating twice we get the expression for $\ddot{q}_{m}$ as

$$
\begin{gathered}
\ddot{q}_{m}=\ddot{q}_{a}+K^{-1}\left[M_{a}\left(q_{a}\right) q_{a}^{[4]}+2 \dot{M}_{a}\left(q_{a}, \dot{q}_{a}\right) q_{a}^{[3]}+\right. \\
\left.\ddot{M}_{a}\left(q_{a}, \dot{q}_{a}, \ddot{q}_{a}\right) \ddot{q}_{a}+\ddot{n}\left(q_{a}, \dot{q}_{a}, \ddot{q}_{a}, q_{a}^{[3]}\right)\right],
\end{gathered}
$$

where $x^{[i]}$ denotes $d^{i} x / d t^{i}$. Adding (1a) to (1b) yields

$$
u=M_{a}\left(q_{a}\right) \ddot{q}_{a}+n\left(q_{a}, \dot{q}_{a}\right)+M_{m} \ddot{q}_{m} .
$$

Inserting (4) in (5) gives

$$
u=\tau\left(q_{a}, \dot{q}_{a}, \ddot{q}_{a}, q_{a}^{[3]}, q_{a}^{[4]}\right),
$$

which shows that the flexible joint model is a flat system with the flat output $q_{a}$. By choosing the states

$$
x=\left[\begin{array}{c}
q_{a} \\
\dot{q}_{a} \\
\ddot{q}_{a} \\
q_{a}^{[3]}
\end{array}\right]=\left[\begin{array}{c}
x_{1} \\
x_{2} \\
x_{3} \\
x_{4}
\end{array}\right],
$$

the system can be expressed in the following state-space form by the use of $(4)-(6)$

$$
\begin{aligned}
\dot{x}_{1} & =x_{2}, \\
\dot{x}_{2} & =x_{3}, \\
\dot{x}_{3} & =x_{4}, \\
\dot{x}_{4} & =f(x)+g(x) u, \\
y & =x_{1},
\end{aligned}
$$


where

$$
\begin{aligned}
f(x)= & -M_{a}^{-1}\left(x_{1}\right) K M_{m}^{-1}\left(M_{a}\left(x_{1}\right) x_{3}+n\left(x_{1}, x_{2}\right)\right)- \\
& M_{a}^{-1}\left(x_{1}\right)\left(K+\ddot{M}_{a}\left(x_{1}, x_{2}, x_{3}\right)\right) x_{3}- \\
& 2 M_{a}^{-1}\left(x_{1}\right) \dot{M}_{a}\left(x_{1}, x_{2}\right) x_{4}- \\
& M_{a}^{-1}\left(x_{1}\right) \ddot{n}\left(x_{1}, x_{2}, x_{3}, x_{4}\right) \\
g(x)= & M_{a}^{-1}\left(x_{1}\right) K M_{m}^{-1} .
\end{aligned}
$$

It is clear that four differentiations of each component of the output $y$ are needed in order for $y_{i}^{[4]}$ to depend directly on $u$, i.e., the relative degree $\nu_{i}=4$. Now, $\Sigma \nu_{i}=4 N$ so the system has full relative degree and no zero dynamics associated with output $y$ (Isidori, 1995; Slotine and $\mathrm{Li}, 1991$ ). Thus the system is fully linearizable by a static feedback control law that can be derived from the controller canonical form (8) as

$$
u=g^{-1}\left(x_{m}\right)\left(v-f\left(x_{m}\right)\right),
$$

where $v$ is a new control signal for the linearized and decoupled system $q_{a}^{[4]}=v$ consisting of $N$ independent chains of four integrators. For tracking control, $v$ can be chosen as

$$
v=q_{a, r}^{[4]}+L\left(x_{r}-x_{m}\right),
$$

where $L \in R^{N \times 4 N}$ is a linear feedback gain matrix, $x_{r}$ are the reference states, and $x_{m}$ are the measurements of the states $x$. The fourth derivative of the reference trajectory must be defined and is denoted $q_{a, r}^{[4]}$. This control law can also be derived by inserting the measured states $x_{m}$ and $v$ in (6) to yield

$$
u=\tau\left(x_{1 m}, x_{2 m}, x_{3 m}, x_{4 m}, q_{a, r}^{[4]}+L_{1}\left(x_{r}-x_{m}\right)\right) .
$$

The derived control law is a combination of feedback and feedforward where the feedback part is dominating and is from now on denoted FL.

A feedforward dominant control law can be expressed as

$$
u=\tau\left(x_{1 r}, x_{2 r}, x_{3 r}, x_{4 r}, q_{a, r}^{[4]}\right)+L_{2}\left(x_{r}-x_{m}\right),
$$

where, ideally in the case of a perfect model, all torque needed for the desired trajectory is computed by feedforward. This control law is denoted FF. Note that for both controllers, in a real implementation, an integral term would be needed to handle model errors and disturbances. For simplicity the integral term is omitted in this study.

The control law (12) gives constant bandwidth of the linearized closed loop for all robot configurations. For constant bandwidth there would be no need for gain scheduling. Gain scheduling is on the other hand probably needed in (13). This can be accomplished by observing that the closed loop dynamics for (12) is given by (8) with $\dot{x}_{4}=-L_{1} x$ and that $L_{2}$ is given by setting $-L_{1} x=f(x)+$ $g(x)\left[-L_{2} x\right]$. If the system is linearized for zero gravity, zero speed we get the configuration dependant feedback gain

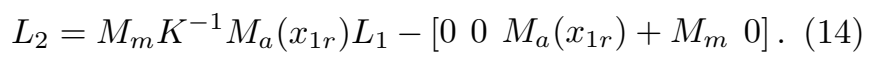

In this way we get approximately the same linear feedback for both control laws which will facilitate the comparison.

The two control laws are illustrated in Figure $2-3$. The control signal $u$ can be described as

$$
u=u_{d, n c}+u_{f f w}+u_{f d b},
$$

where $u_{d, n c}$ is the torque for decoupling and nonlinear cancellation, $u_{f f w}$ is the feedforward torque and $u_{f d b}$ is the torque from the linear feedback controller. For FF, $u_{d, n c}=0$.

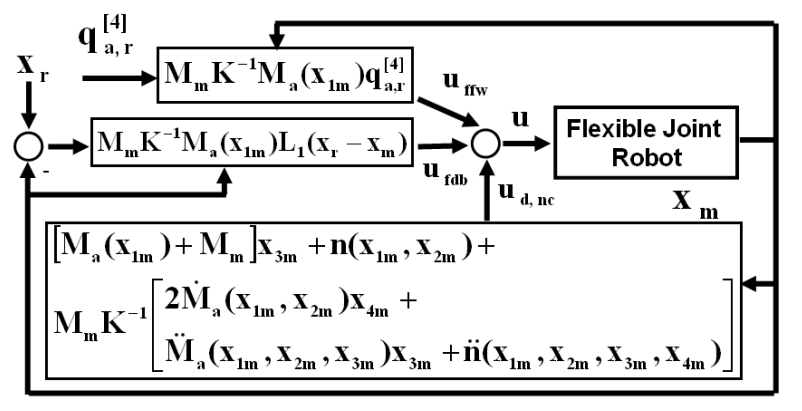

Fig. 2. Feedback Linearization Control Law (FL)

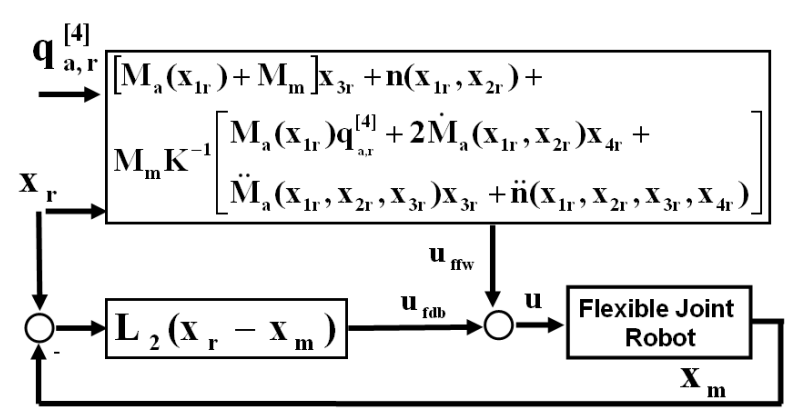

Fig. 3. Feedforward Control Law (FF)

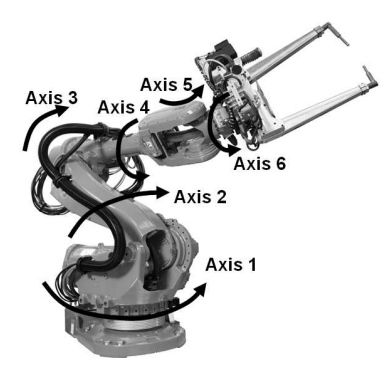

Fig. 4. IRB6600 from ABB equipped with a spot welding gun

\section{SIMULATION STUDY}

The robot model described in Section 2 is simulated with the controller structures from Section 3 . The model parameters used are typical for the three first axes of a large industrial robot with a payload capacity of $150 \mathrm{~kg}$. One example of such a robot is shown in Figure 4 and the frequency response function of the linearized model in one configuration is shown in Figure 5. For simplicity, the gravitational constant is set to zero.

The requirement specification illustrates a typical requirement for a dispensing application, e.g., gluing inside a car body, and is stated as follows:

- The programmed path should be followed by an accuracy of $1 \mathrm{~mm}$ (maximum deviation) at an acceleration of $8 \mathrm{~m} / \mathrm{s}^{2}$ and a speed of $0.5 \mathrm{~m} / \mathrm{s}$.

- The specification above must be fulfilled for model errors in the user load mass by $\pm 20 \%$ and errors in the gear-box stiffness of $\pm 20 \%$.

- The test path is a circular path with radius $25 \mathrm{~mm}$.

The smooth cartesian trajectory reference $Z_{r}(\theta(t))$ is compared to the obtained robot trajectory $Z(\eta(t))$, where $\theta$ and $\eta$ are time dependant scalar path parameters. Instead of using the tracking error $\left|Z_{r}(t)-Z(t)\right|$, the path accuracy 


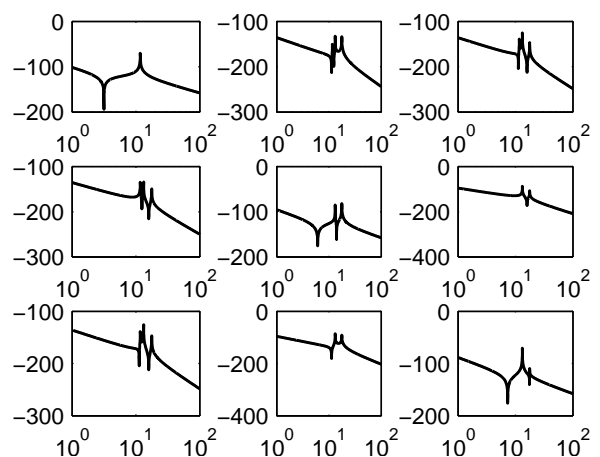

Fig. 5. The frequency response function from motor torque to motor speed. Magnitude in $[\mathrm{dB}]$ and frequency in $[\mathrm{Hz}]$.
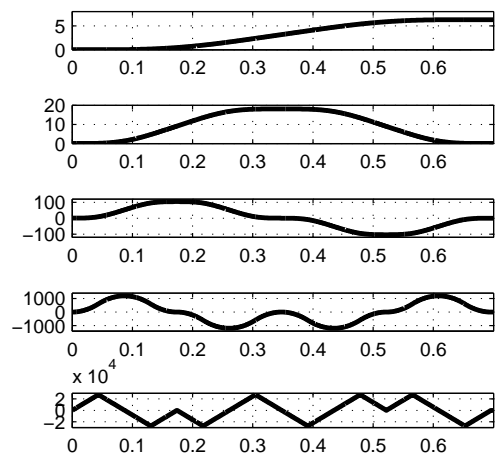

Fig. 6. The circular angle $Q$ and its derivatives

is measured by the path-following error. The tracking error will indicate error for, e.g., a small time delay between $Z_{r}$ and $Z$. Therefore, the path-following error is preferred as an accuracy measure and is also used in ISO (1998). The maximum path-following error is here computed as

$$
e=\max _{\eta \in Z}\left(\min _{\theta \in Z_{r}}\left(\left|Z_{r}(\theta)-Z(\eta)\right|\right)\right) \text {. }
$$

The circle computed in polar coordinates, radius $r$, angle $\mathrm{Q}$, by discrete integration of $Q^{[5]}(t)$ and thus the path is $\mathcal{C}^{4}$ as shown in Figure 6. The link angle reference $q_{r}$ is then computed using inverse kinematics and the required derivatives are calculated without delay.

\subsection{Nominal Performance}

In this section, the performance of feedback linearization and feedforward control is evaluated for the nominal case, i.e., no model errors. The discrete implementation is straightforward as the control laws, including all model derivatives $\left(\dot{M}_{a}, \ddot{M}_{a}\right.$, and $\left.\ddot{n}\right)$, are expressed as algebraic equations. The robot model is simulated as a continuous system with a zero-order hold at the output. Figure 7 shows an example of a simulated path with a path error of $4 \mathrm{~mm}$. Simulations were performed for the nominal system with different sample times and different bandwidths of the linearized closed loop. Two methods for computing the feedback gain matrix $L_{1}$ were used, $L Q$ optimal control (Anderson and Moore, 1990) and pole placement. For feedforward control, $L_{2}$ is then computed from $L_{1}$ according to (14). The controller is implemented with a fixed sample rate. The measured variables were filtered with a second order continuous anti-alias filter of Butterworth type, with a bandwidth equal to the Nyquist frequency. The bandwidth of of the linearized closed loop

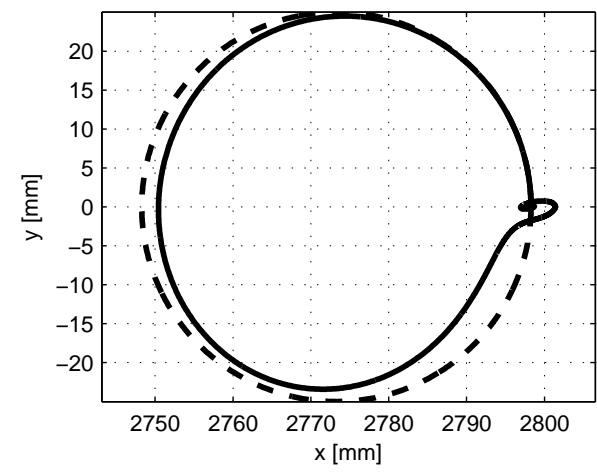

Fig. 7. An example of the circular reference path (dashed) and robot path (solid)

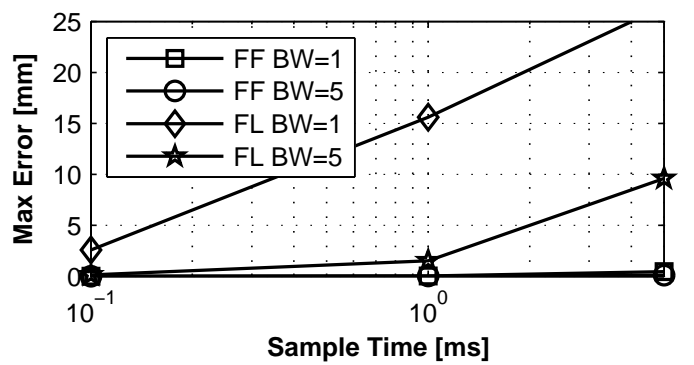

Fig. 8. Path error vs. sample time for feedback linearization (FL) and feedforward (FF) using an $L Q$ linear controller. Bandwidth (BW) of the linearized closed loop is $1 \mathrm{~Hz}$ and $5 \mathrm{~Hz}$.

is indicated by the absolute value of dominant closed loop poles. Figure 8 - 9 show the path errors at different sample times and at two different bandwidths. The linear $L Q$-controller clearly causes problems when feedback linearization is used. Hence, in the remaining simulations, pole placement is used for the linear controller design. The design of the linear controller has not been further analyzed since the objective of this work is to compare the nonlinear controller concepts. Figure 10 shows the path errors for feedback linearization when different nominal motor inertias are used. The nominal motor inertias in all other simulations are $200 \mathrm{kgm}^{2}$, expressed on the link side. The performance of feedback linearization clearly depends on the motor inertia. Other simulations also show that the path error increases for feedback linearization if the anti-alias filtering of the measured variables is increased, i.e., the bandwidth is decreased or the order of the filter is increased. Feedforward control does not show the same sensitivity in this respect.

\subsection{Robust Performance}

The uncertain system, according to the requirement specification, was then simulated with a set of 20 models. The models were a combination of extreme values for the uncertain parameters and some random systems inside the uncertainty description. The result for one simulation example is shown in Figure 11 and an example of the resulting path error is shown in Figure 12. All simulations are summarized Table 1. 


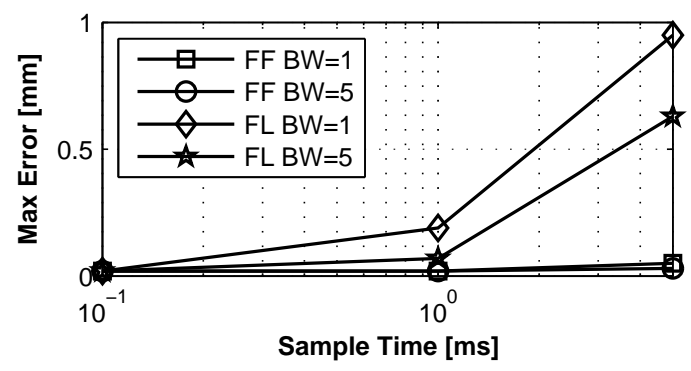

Fig. 9. Path error vs. sample time for feedback linearization (FL) and feedforward (FF) using a pole placement linear controller. Bandwidth (BW) of the linearized closed loop is $1 \mathrm{~Hz}$ and $5 \mathrm{~Hz}$.

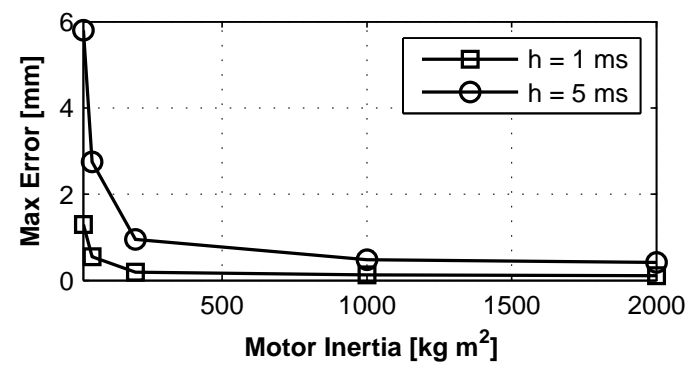

Fig. 10. Path error vs. motor inertia for feedback linearization (FL) using a pole placement linear controller. Bandwidth of the linearized closed loop is $1 \mathrm{~Hz}$. Two different sample times (h) are used. Feedforward control (not shown) yields an error less than $0.2 \mathrm{~mm}$ in all cases.

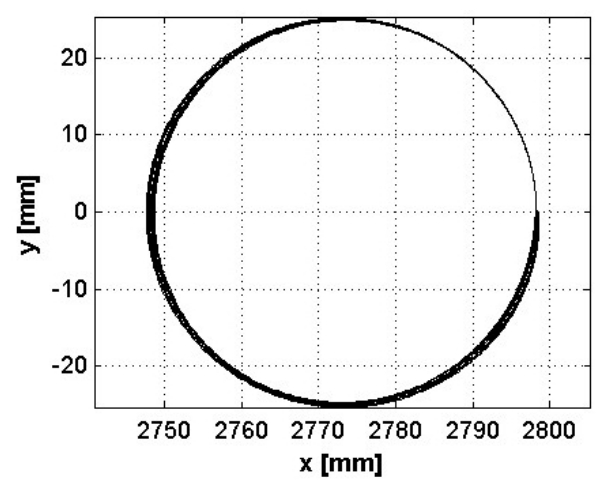

Fig. 11. Simulation of uncertain systems for feedback linearization control. Sample time is $1 \mathrm{~ms}$ and linearized closed loop bandwidth is $1 \mathrm{~Hz}$.

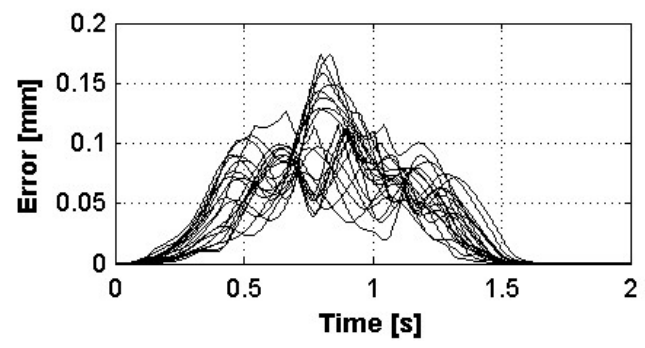

Fig. 12. Path error of uncertain systems for feedback linearization control. Sample time is $1 \mathrm{~ms}$ and linearized closed loop bandwidth is $5 \mathrm{~Hz}$.
Table 1. Summary of simulation results for circular path (radius $25 \mathrm{~mm}$ ) and the uncertain system.

\begin{tabular}{|l|l|l|l|}
\hline \hline Sample Time $[\mathrm{ms}]$ & Bandwidth $[\mathrm{Hz}]$ & Method & Error $[\mathrm{mm}]$ \\
\hline 1 & 1 & FF & 0.56 \\
\hline 1 & 1 & FL & 0.70 \\
\hline 1 & 5 & FF & 0.14 \\
\hline 1 & 5 & FL & 0.17 \\
\hline 5 & 5 & FF & 0.24 \\
\hline 5 & 5 & FL & 1.10 \\
\hline
\end{tabular}

\subsection{Discussion}

From the simulation results, the following conclusions are drawn:

- FL requires higher sample rate than FF.

- Low sample rate can to some extent be compensated by high bandwidth of the linearized closed loop.

- If these requirements are fulfilled, the nominal and robust performance of FL is similar to FF. However, in this simulation study, FF always yields a better result.

- The value of the motor inertia is critical for FL.

- Measurement filtering, and hence time delay, reduces the performance more for FL than for FF.

- The design of the linear feedback controller is critical for good performance. FL is more sensitive in this respect.

To understand these results, it is helpful to consider the different torque components in (15). The result can now be explained as follows, the nonlinear cancellation and decoupling performed by FL in an ideal continuous world does not work in the same way for discrete time. Thus, the inevitable delay of $u_{d, n c}$, caused by the discrete implementation, causes path errors if the sample time is too high. Increasing the bandwidth of the linearized closed loop will compensate for the delay in $u_{d, n c}$ by a correcting torque in $u_{f d b}$. For FF and small model errors, $u_{f f w}$ is dominating and the errors are small even for a moderate sample rate and measurement filtering. In FL, the amount of torque in $u_{d, n c}$ and $u_{f f w}$ depends on the numerical values of the model parameters. This explains why an increase in motor inertia decreases the error. From Figure 2 it is seen that increased motor inertia increases $u_{f f w}$ relative to $u_{d, n c}$.

In feedback linearization of a rigid manipulator, i.e., the classical computed torque algorithm, $u_{d, n c}$ consists of the slow varying gravity torque and the speed dependent torques. The fastest varying torque is the acceleration dependant torque which is included in $u_{f f w}$. In this case the problems described above should be considerably smaller than in the case of a flexible joint robot where $u_{d, n c}$ also depends on the fast varying acceleration and jerk. To understand the different behavior of feedback linearization for different systems, consider the algorithm applied to a rigid one-axis manipulator with no friction or gravity, i.e., a double integrator. If the model inertia is correct, all required torque will be included in $u_{f f w}$ and $u_{d, n c}$ is zero. $\mathrm{FL}$ and FF is the same in this case.

To summarize, time delays caused by discrete implementation and filtering causes the linearization and decoupling to be only partial. The incomplete decoupling can, e.g., be analyzed by linearizing the system around position $q_{a 0}$, and applying the feedback linearization (including 
measurement filtering) to the linearized and discretized system. The system is then decoupled by using

$$
u=\left(M_{a}\left(q_{a 0}\right)+M_{m}\right) \ddot{q}_{a}+M_{m} K^{-1} M_{a}\left(q_{a 0}\right) v .
$$

This deviation from the ideal decoupled system must be handled by the linear controller, which therefore is critical for the resulting performance. Of course there is a limit of the achievable feedback bandwidth. Unmodeled dynamics, model errors, measurement noise and the fact that perfect complete state measurements are unachievable also limit the bandwidth. State estimation, noise filtering, and computational delay also add to the total delay. The sample rate and time delays can theoretically be very small but in practise there are limitations. The requirement on computational power would be enormous for a six-axes manipulator. Computation of model derivatives by numerical differentiation would reduce the number of operations, but would on the other hand also increase the delay.

\section{SUMMARY}

In this paper, the discrete implementation of feedback linearization for flexible joint robots has been investigated in a simulation study concerning high speed tracking. Feedback linearization has been compared with feedforward control. Perfect state measurements have been assumed. With this assumption, the result is that typical industrial robot requirements can be fulfilled with both concepts. Another result is that feedforward control gives better performance and puts less requirements on the controller and sensor hardware. The design of the linear controller is found to be critical for the resulting performance. The feedback controller is needed for robustness, stabilization and disturbance rejection. The partial linearization and decoupling, caused by the discrete implementation, increases the requirements on the feedback controller. Of course, there are more ways to combine feedback and feedforward than the two concepts studied in this article. Given the flat expression of the control signal (6) there are many ways to insert the measured states, the references states and the amplified state errors into the equation. Thus, how the optimal feedforward/feedback controller for an elastic robot manipulator should be designed is still an open question.

\section{REFERENCES}

A. Albu-Schäffer and G. Hirzinger. State feedback controller for flexible joint robots: A globally stable approach implemented on DLR's light-weight robots. In Proceedings of the 2000 IEEE/RSJ International Conference on Intelligent Robots and Systems, 2000.

C. An, C. Atkeson, and J. Hollerbach. Model-Based Control of a Robot Manipulator. The MIT press, Cambridge, Massachusetts, 1988.

B. Anderson and J. Moore. Optimal Control: Linear Quadratic Methods. Prentice-Hall, 1990.

D. Bernstein. On bridging the theory/practise gap. IEEE Control Systems Magazine, 19(6):64-70, 1999.

F. Caccavale and P. Chiacchio. Identification of dynamic parameters and feedforward control for a conventional industrial manipulator. Control Eng. Practice, 2(6): 1039-1050, 1994.

A. De Luca. Feedforward/feedback laws for the control of flexible robots. In Proceedings of the 2000 IEEE International Conference on Robotics and Automation, pages 233-240, San Francisco, CA, April 2000.
A. De Luca. Dynamic control of robots with joint elasticity. In Proceedings of the 1988 IEEE International Conference on Robotics and Automation, pages 152-158, Philadelphia, PA, 1988.

A. De Luca and P. Lucibello. A general algorithm for dynamic feedback linearization of robots with elastic joints. In Proceedings of the 1998 IEEE International Conference on Robotics and Automation, pages 504-510, Leuven, Belgium, May 1998.

A. Isidori. Nonlinear Control Systems. Springer-Verlag, 1995.

ISO. ISO 9283:1998, manipulating industrial robots - performance criteria and related test methods. www. iso.org, 1998.

K. P. Jankowski and H. Van Brussel. An approach to discrete inverse dynamics control of flexible-joint robots. IEEE Transactions on Robotics and Automation, 8(5): 651-658, October 1992.

T. R. Kane and D. A. Levinson. Dynamics: Theory and Applications. McGraw-Hill Publishing Company, 1985.

M. Lesser. The Analysis of Complex Nonlinear Mechanical Systems: A Computer Algebra assisted approach. Singapore: World Scientific Series on Nonlinear Science, 2000.

S. Nicosia and P. Tomei. On the feedback linearization of robots with elastic joints. In Proceedings of the $27 n d$ Conference on Decision and Control, pages 180-185, Austin, Texas, December 1988.

M. V. Nieuwstadt and R. Murray. Real-time trajectory generation for differentially flat systems. International Journal of Robust and Nonlinear Control, 8(11):9951020, 1998.

D. Ridgely and M. McFarland. Tailoring theory to practise in tactical missile control. IEEE Control Systems Magazine, 19(6):49-55, 1999.

P. Rouchon, M. Fliess, J. Lévine, and P. Martin. Flatness, motion planning and trailer systems. In Proceedings of the 32nd Conference on Decision and Control, pages 2700-2705, San Antonio, Texas, December 1993.

V. Santibanez and R. Kelly. PD control with feedforward compensation for robot manipulators: analysis and experimentation. Robotica, 19:11-19, 2001.

L. Sciavicco and B. Siciliano. Modelling and Control of Robot Manipulators. Springer, 2000.

J.-J. Slotine and W. Li. Applied Nonlinear Control. Prentice Hall, 1991.

M. Spong, S. Hutchinson, and M. Vidyasagar. Robot Modeling and Control. Wiley, 2006.

M. W. Spong. Modeling and control of elastic joint robots. Journal of Dynamic Systems, Measurement, and Control, 109:310-319, December 1987.

K. Åström. The future of control. Modeling, Identification and Control, 15(3):127-134, 1994.

M. Thümmel, M. Otter, and J. Bals. Control of robots with elastic joints based on automatic generation of inverse dynamic models. In Proceedings of the 2001 IEEE/RSJ International Conference in Intelligent Robots and Systems, pages 925-930, Maui, Hawaii, USA, October 2001.

E. Wernholt and S. Moberg. Frequency-domain gray-box identification of industrial robots. In Proc. 17th IFAC World Congress, Seoul, Korea, July 2008. 


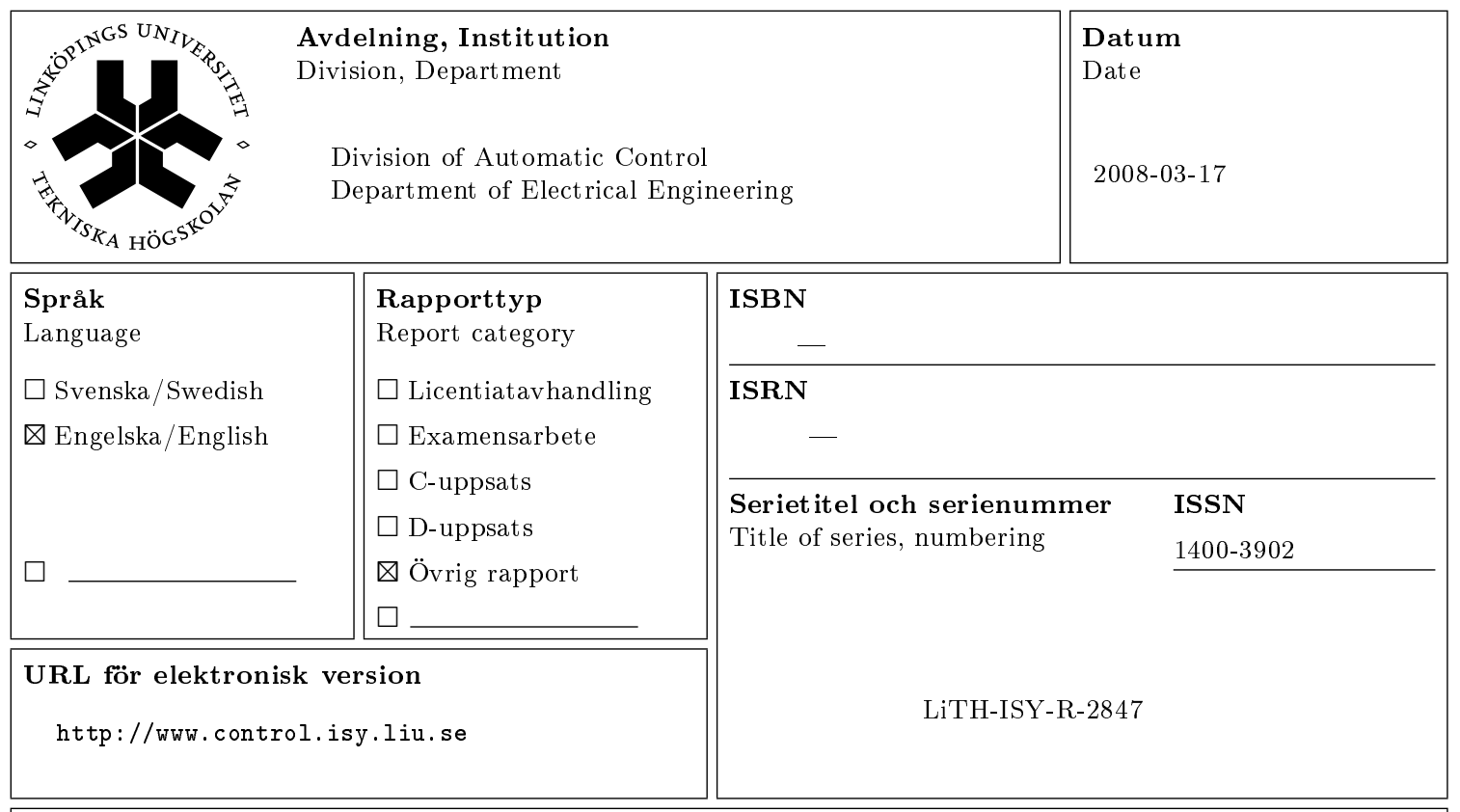

Titel On Feedback Linearization for Robust Tracking Control of Flexible Joint Robots

Title

Författare Stig Moberg, Sven Hanssen

Author

Sammanfattning

Abstract

Feedback linearization is one of the major academic approaches for controlling flexible joint robots. This contribution investigates the discrete-time implementation of the feedback linearization approach for a realistic three-axis robot model. A simulation study of high speed tracking with model uncertainty is performed. It is assumed that full state measurements of the linearizing states are available. The feedback linearization approach is compared to a feedforward approach. 